\title{
BMJ Open Economic evaluation of the Midwifery Initiated Oral Health-Dental Service programme in Australia
}

\author{
Kathy W Tannous (D) , ${ }^{1}$ Ajesh George (D) ,2,3 Moin Uddin Ahmed (D) , ${ }^{4}$ \\ Anthony Blinkhorn, ${ }^{3}$ Hannah G Dahlen (D) , ${ }^{5,6}$ John Skinner (1) , 7 Shilpi Ajwani, ${ }^{3,8}$ \\ Sameer Bhole, ${ }^{3,8}$ Albert Yaacoub, ${ }^{9}$ Ravi Srinivas, ${ }^{2,3,10}$ Maree Johnson (i) ${ }^{6,11}$
}

To cite: Tannous KW, George A, Ahmed MU, et al. Economic evaluation of the Midwifery Initiated Oral HealthDental Service programme in Australia. BMJ Open 2021;11:e047072. doi:10.1136/ bmjopen-2020-047072

- Prepublication history for this paper is available online. To view these files, please visit the journal online (http://dx.doi org/10.1136/bmjopen-2020047072).

Received 18 November 2020 Accepted 19 July 2021

\section{Check for updates}

(c) Author(s) (or their employer(s)) 2021. Re-use permitted under CC BY-NC. No commercial re-use. See rights and permissions. Published by BMJ.

For numbered affiliations see end of article.

\section{Correspondence to}

Prof Ajesh George;

a.george@westernsydney. edu.au

\section{ABSTRACT}

Objectives To critically evaluate the cost-effectiveness of the Midwifery Initiated Oral Health-Dental Service ( $\mathrm{MIOH}-$

DS) designed to improve oral health of pregnant Australian women. Previous efficacy and process evaluations of MIOH-DS showed positive outcomes and improvements across various measures.

Design and setting The evaluation used a cost-utility model based on the initial study design of the MIOH-DS trial in Sydney, Australia from the perspective of public healthcare provider for a duration of 3 months to 4 years.

Participants Data were sourced from pregnant women $(n=638)$, midwives $(n=17)$ and dentists $(n=3)$ involved in the $\mathrm{MIOH}$ trial and long-term follow-up.

Cost measures Data included in analysis were the cost of the time required by midwives and dentists to deliver the intervention and the cost of dental treatment provided. Costs were measured using data on utilisation and unit price of intervention components and obtained from a micro-costing approach.

Outcome measures Utility was measured as the number of Disability Adjusted Life Years (DALYs) from health-benefit components of the intervention. Three cost-effectiveness analyses were undertaken using different comparators, thresholds and time scenarios.

Results Compared with current practice, midwives only intervention meets the Australian threshold (A\$50 000) of being cost-effective. The midwives and accessible/ affordable dentists joint intervention was only 'costeffective' in 6 months or beyond scenarios. When the midwife only intervention is the comparator, the midwife/ dentist programme was 'cost-effective' in all scenarios except at 3 months scenario.

Conclusions The midwives' only intervention providing oral health education, assessment and referral to existing dental services was cost-effective, and represents a low cost intervention. Midwives' and dentists' combined interventions were cost-effective when the benefits were considered over longer periods. The findings highlight short and long term economic benefits of the programme and support the need for policymakers to consider adding an oral health component into antenatal care Australia wide.

Trial registration number ACTRN12612001271897; Post-results.

\section{Strengths and limitations of this study}

- This is the first economic evaluation undertaken to determine if an antenatal oral health intervention led by midwives (Midwifery Initiated Oral Health-Dental Service) is cost-effective for health service administrators and policy planners in the Australian context.

- The Disability Adjusted Life Years measure was used instead of the Quality Adjusted Life Years which may have limitations to measure effectiveness.

- The study findings are sensitive to the probability of timely dental treatment.

- The study did not consider any health benefits for the children following birth as these data were not available.

\section{INTRODUCTION}

Oral health is an integral part of antenatal care with a dental check-up being recommended early in pregnancy. ${ }^{1}$ Poor oral health is aggravated during pregnancy by hormonal changes, vomiting, heartburn and nausea raising the risk of women suffering from the common conditions of dental decay, gum disease and dental erosion. ${ }^{2}$ Promoting oral health and providing dental care can improve oral hygiene, help prevent dental disease ${ }^{3}$ and delay the risk of mothers transmitting decay-causing bacteria to their children, a known cause of early-childhood caries. ${ }^{4} 5$ Furthermore, raising oral health awareness during pregnancy can foster good preventive oral hygiene practices and dietary behaviours in children particularly around the consumption of sugar-sweetened beverages which is a key risk factor for early-childhood caries. ${ }^{67}$

Despite its importance, in an Australian context less than half of the pregnant women seek dental care, largely due to cost barriers. ${ }^{8-10}$ Oral health issues are often compounded in low socioeconomic households by the costs associated with seeking dental treatment in Australia's user payer 
dental system and low rates of extras cover for dental treatment through private health insurance. ${ }^{11-13}$ Poor dental attendance, and resulting poor oral health, are closely associated with income, with only $22 \%$ of lowincome households having a favourable dental visiting pattern to dentists compared with $56 \%$ of higher-income households. ${ }^{14}$

Dental care in Australia is provided predominantly by private dental providers to individuals with or without private health insurance with dental cover. ${ }^{15}$ Publiclyfunded oral and dental health services operated by the states and territories offer free or subsidised services but these vary across jurisdictions. ${ }^{15}$ Adults seeking to access public dental care must hold an Australian Government issued concession card such as Healthcare Card Pensioner Concession Card or the Commonwealth Seniors card. These are issued to persons receiving government benefit payments including aged pensions, unemployed job seekers, some students, carers and people raising children. ${ }^{15}$ Within the eligible population for public dental care, some states and territories identify target populations for priority care. These patients are usually not placed on a waiting list, but are immediately scheduled for a dental care appointment. In the Australian States of Victoria and Tasmania, for example, pregnant women are in the priority or target populations for public dental services and are provided the next available appointment usually within 4 weeks. ${ }^{16}$ Other states and territories, such as New South Wales (NSW), do not assign urgent priority to pregnant women and they can wait up to 3 months for a dental appointment. ${ }^{17}$

Aside from costs, other important barriers for pregnant women seeking to access dental services in Australia include lack of awareness about the importance of oral health and misconceptions about oral health during pregnancy. ${ }^{10}$ Women are often unaware of the impact that poor oral health can have on pregnancy and infant outcomes. ${ }^{8-10}$ Further, many have concerns regarding the safety of dental treatment and radiographs during pregnancy and feel that dentists may be unwilling to treat them while they are pregnant. ${ }^{8918}$ Exacerbating the situation is the limited emphasis being placed on oral health by antenatal care providers in Australia due to their limited knowledge and training in this area. ${ }^{19} 20$ A recent survey of antenatal care providers in NSW found that very few $(16 \%-21 \%)$ discussed the importance of oral health with pregnant patients or advised them to visit a dentist. ${ }^{19}$

To address current barriers and improve the oral health of pregnant women in Australia the study investigators developed the Midwifery Initiated Oral HealthDental Service (MIOH-DS) programme. ${ }^{21}$ As part of the programme midwives, who are the main healthcare providers for pregnant women in Australia, ${ }^{22}$ were trained to incorporate oral health guidelines into their practice and provide pregnant women with oral health education, risk assessment and referrals using existing referral pathways $(\mathrm{MIOH})$ (private dentists, health funds, public dental services with eligibility criteria) or priority referrals to public dental services irrespective of eligibility criteria (MIOH-DS). ${ }^{23}$ The programme was comprehensively evaluated through a large, multicentre randomised controlled trial in NSW involving pregnant women who used public, private and healthfund dental services. ${ }^{23}$ The results showed a significant improvement in the uptake of dental services, oral health outcomes, quality of oral health and level of oral health knowledge among pregnant women who received the MIOH-DS intervention. ${ }^{23}$ There was also a significant improvement in the oral health knowledge and confidence of midwives to promote oral health. ${ }^{25}$ In addition, a process evaluation demonstrated that the programme was both acceptable and feasible for midwives, pregnant women and dentists. ${ }^{26-28}$ An economic evaluation of the MIOH-DS programme is the next important step in determining whether this programme should be implemented more widely.

Economic evaluations of healthcare are now expected in an increasingly fiscally challenged health budget. Healthcare costs continue to grow faster than the gross domestic product (GDP). ${ }^{29}$ The USA has the highest health expenditure in the world at over $17 \%$ of GDP in $2017 .^{30}$ In Australia, the health expenditure in the same period was $9.2 \%$ of GDP, which is higher than the Organisation for Economic Co-operation and Development median of $8.2 \% .^{31}$ Dental expenditure by Australia's state and national government totalled $\mathrm{A} \$ 2.4$ billion in $2017 / 2018$, approximately $2 \%$ of the total health expenditure.$^{29}$ Cost-effectiveness analysis aims to support policy decision-making through the allocation of scarce health resources. Midwifery care has increasingly become the focus of economic evaluations with findings demonstrating cost-effectiveness with midwife led models of care $^{32} 33$ and practices. ${ }^{34}$ Evaluating cost-effectiveness of the MIOH-DS programme is an important addition to this growing body of work.

\section{Objectives}

The aim of this study was to evaluate the cost-effectiveness of $\mathrm{MIOH}$ and MIOH-DS programmes compared with current practice.

\section{METHODS \\ Design and setting}

Data were obtained from a randomised controlled trialintervention group from three metropolitan public hospitals in the Greater Sydney region. The initial trial has been detailed elsewhere..$^{21}$ It was not possible to include patients in the design, conduct, reporting, or dissemination plans of our research.

\section{Participants}

Recruitment $(\mathrm{n}=638)$ and follow-up $(\mathrm{n}=476)$ of the trial occurred between November 2012-October 2015. A long-term follow-up of the participants and their children $(\mathrm{n}=204)$ was undertaken between the years 2016-2018 
the results of which will be reported elsewhere. ${ }^{35}$ The participants were pregnant women, from 12 to 20 weeks, who attended for their first antenatal visit and met the criteria of the study as detailed elsewhere. ${ }^{21}$

Women in the Control Group (CG, n=168) were provided with written information on oral health at baseline. All women in the study who were perceived to have dental problems were referred to a study dentist after their pregnancy was completed.

Those in Intervention Group 1 (IG1, n=152) (MIOH) received the same services as those in the control group, plus:

1. Were provided with information verbally on oral health and pregnancy,

2. Had their oral health risk assessed by midwives using a validated Maternal Oral Screening (MOS) tool, ${ }^{24}$ and

3 . Were referred to existing dental services (private, public if eligible or health fund clinic) for further diagnosis and treatment if rated as at risk for poor oral health. Women in Intervention Group 2 (IG2, n=156) (MIOHDS) received the same information and assessments as those in IG1, but were also referred to a study dentist at a public dental clinic for a free dental assessment and treatment to maintain a functional and infection-free oral cavity.

\section{Intervention}

Prior to commencement of the trial, all participating midwives $(n=17)$ completed a specific oral health professional development training programme through the Australian College of Midwives. ${ }^{25}$ In addition, the three study dentists employed for the trial participated in a 2-hour education workshop where they were trained by an experienced dental clinician to follow a standardised protocol during the programme.

The components of the intervention were:

1. Education and training of midwives in providing oral health education, screening (using the MOS tool) ${ }^{24}$;

2. Education of the three dentists attached to the study on a standardised dental treatment protocol for pregnant women;

3. Provision of dental check-ups by dentists for the IG2 at baseline and follow-up and for all participants at follow-up; and

4. Provision of standardised, publicly funded dental treatment for women in IG2 as needed during the period of their pregnancy, provided it was safe to undertake such treatment.

\section{Patient and public involvement}

Initial exploratory work $^{9}{ }^{10}$ with pregnant women regarding their perceptions of oral healthcare and the potential role of midwives informed the development of the research question, outcome measures and study design. Pregnant women were not involved in the recruitment and conduct of the study. As part of the broader study a process evaluation reviewed participants' experience of taking part in the randomised controlled trial. ${ }^{28}$
A summary of the study results was disseminated to all interested pregnant women who took part in the trial.

\section{Perspective of the study and comparators}

The perspective considered in this study is public healthcare provider. Three cost-effectiveness analyses were undertaken with different comparators depending on the scenarios, which were:

1. MIOH-DS (IG2) as intervention group and current practice (CG) as comparator group

2. MIOH (IG1) as intervention group and current practice $(C G)$ as comparator group.

3. MIOH-DS (IG2) as intervention group and $\mathrm{MIOH}$ (IG1) as comparator group.

Data were collected on the programme participants (pregnant women) included:

- Baseline information provided through self-completed questionnaires by the pregnant women (12-20 weeks gestation) in the three groups. This information included basic demographics (mean age, country of birth, main language spoken, tertiary education, marital status, socioeconomic status, employment, smoking and other substance use, access to private health insurance and concession cards) and dental information such as self-reported dental problems and dental visits in the last 12 months. ${ }^{23}$

- Midwives' use of the MOS tool in IG1 and IG2.

- Pre-assessment forms completed by the dentists at the initial examination of MIOH-DS and of all participants in the three groups of IG1, IG2 only and CG at completion (28-40 weeks gestation).

- Post-trial information was provided through selfcompleted questionnaires by pregnant women (28-40 weeks gestation). The information collected was similar to the baseline information.

- Medical record number access was obtained for maternal and infant morbidity and mortality.

\section{Resources and costs}

Information on costs was collected at the different stages for the intervention groups. This included the costs of developing and piloting the MIOH-DS intervention including all costs associated with workbooks, screening tools, brochures and production of a DVD. These are capital costs and were treated as sunk costs of the programme. The sunk costs were not included in the study. The costs of using the MIOH instrument by the midwives were assessed based on the time required to evaluate the pregnant women and average midwife salary.

The costs of training the dentists together with the treatments they provided for the women were based on the types of treatment, the time usually taken and the average income for a public dentist. Costs were obtained from a micro-costing approach. The analysis only included cost of time required by midwives and dentists to deliver the intervention and the cost of dental treatment provided.

The outputs for the different groups were calculated from the self-complete questionnaires, the midwives' 
Table 1 Assigned DALY weight to different oral health problems

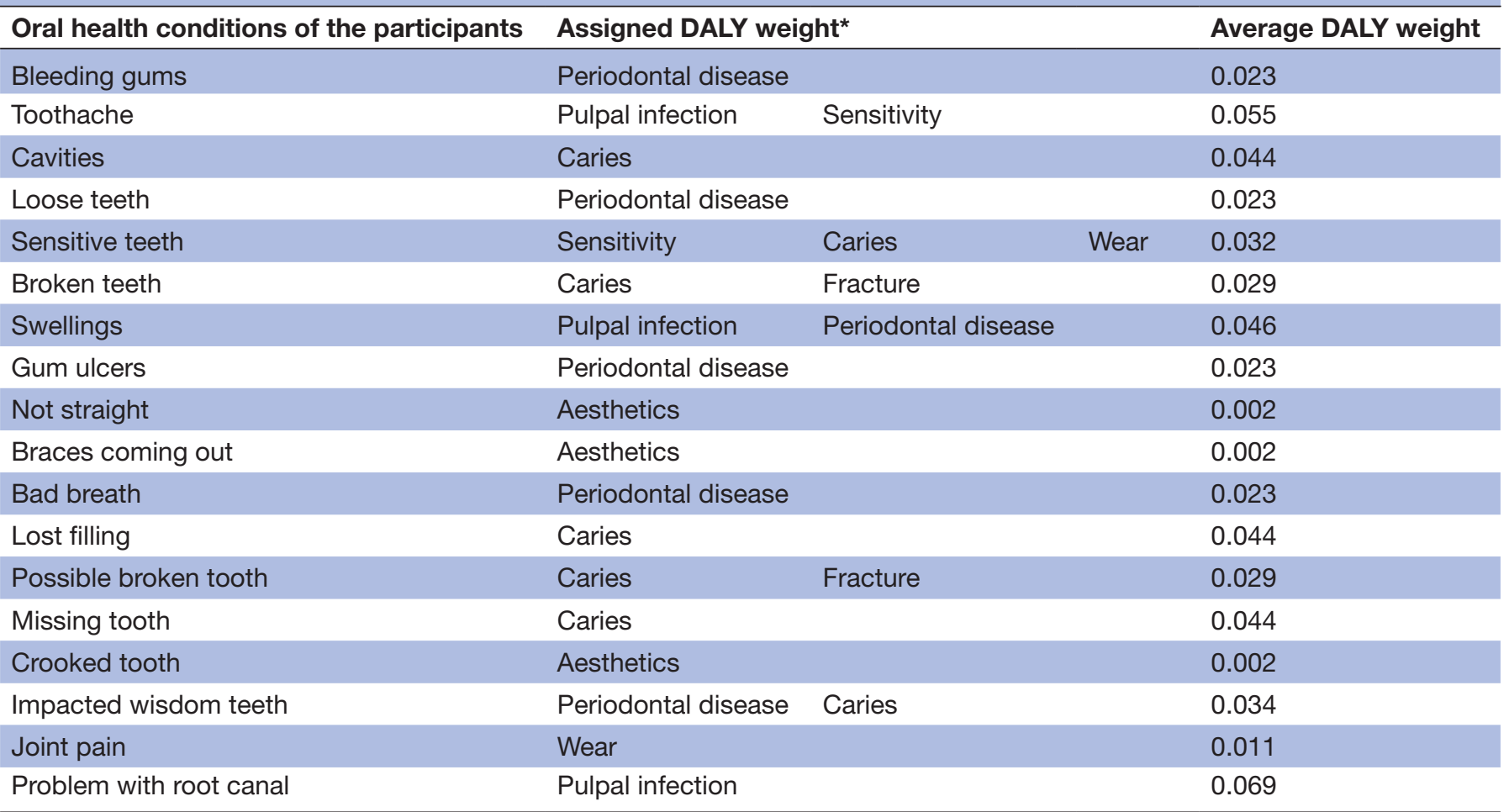

${ }^{*}$ The oral health conditions of the women detailed in the surveys were matched to the Brennan and Spencer ${ }^{36}$ paper and direction was obtained from oral health clinicians for matching and assigning of average DALY weights.

DALY, Disability Adjusted Life Year.

assessments and the dental clinics' assessments and treatments in the different time periods. Stata V.14 and TreeAge Pro were used for the statistical analysis and costeffectiveness analysis, respectively.

Model choice, assumptions and analytical methods

In this study, a decision analytical model was used to perform a cost-utility analysis to assess the desirability of the MIOH-DS programme. The decision tree model was employed in this respect. The components of both cost and health benefits of the programme were defined in the initial study. The costs were measured by collecting and processing data on the utilisation and unit price of these components. The utility was measured using Disability Adjusted Life Years (DALYs) through the use of data on the health-benefit components of the intervention. The oral health conditions of the women detailed in the surveys were matched to the Brennan and Spencer ${ }^{36}$ oral health average DALY weight following direction from oral health clinicians, elaborated in table 1 . The duration used in the analysis ranged from 3 months to 4 years with testing using these periods.

\section{Calculation of costs}

For those in IG1, the programme was delivered by midwives. It was estimated that the additional time required by a midwife (first year award rate) to carry out education, assessment and referral functions was $5 \mathrm{~min}$, with a cost of A $\$ 3.60$. The cost of this intervention was clearly minimal.
Pregnant women in IG2 were also referred to a dentist and needed on average three dental visits to have a functional and infection-free oral cavity. The average cost per dental visit was $\mathrm{A} \$ 236$ which was calculated using dental item numbers collected and costed (from the trial) using the government fee schedule ${ }^{37}$ which includes the salaries of the dental team (dentist and dental assistant) and treatment provided. The total average cost for each pregnant woman to receive the MIOH-DS programme was $\mathrm{A} \$ 711.60(\mathrm{~A} \$ 708+\mathrm{A} \$ 3.60)$. We assume these costs occur once during the period of cost-effectiveness analysis. Costs' calculations were based on the year 2019 for all components.

\section{Calculation of DALYs}

DALYs has two components: years of life lost (YLL) and years of life with disability (YLD). It was assumed that there was no premature death due to the complication of different oral health problems. Therefore, DALYs will be equal to YLDs in our case. To calculate the YLD, the following formula was used:

$$
\mathrm{DALY}=\mathrm{YLD}=\frac{\sum \text { disability weight } \times \text { duration }}{1+\text { discount rate }}
$$

To determine the disability weight, the self-reported MOS tool that was included in the post-trial questionnaire ${ }^{2124}$ was used to determine the dental problems that the participants' had during their pregnancy. This was 
matched with the DALY weight following the methodology described by Brennan and Spencer. ${ }^{36}$

To determine duration, the long-term follow-up survey that was undertaken between 2016-2018 that included the self-reported validated MOS tool ${ }^{24}$ was used and 2018 was used as the final date of the evaluation. In cases where the dates were not available, the average duration of those who had their date mentioned was used. Different scenarios were created that assumed different durations ranges from 3 months to 4 years. In other words, these different lengths can be assumed as disability-free period for the following cases:

- If any treatment is done in case of occurrence of oral health problems during the pregnancy period.

- If women are found without any oral health complication during the pregnancy period.

On the other hand, these periods are assumed to be a period with continued oral health disability for those women who had oral health complications during the pregnancy and did not receive any treatment during the survey period. As the cost of the interventions occurred only once, the health outcomes were discounted by 5\% as per the Australian Pharmaceutical Benefits Advisory Committee guidelines on health technology assessments. ${ }^{38}$ One-way analysis of variance was used to compare mean outcomes between groups.

\section{RESULTS}

Participants were randomised on all main demographic measures and there were no significant differences between groups (table 2).

The incremental cost-effectiveness ratio (ICER) is used to compare, with the Australian threshold value of $A \$ 50$ $000^{38}$ to make a decision on the cost-effectiveness of the intervention. The ICERs are also compared with other Australian thresholds as well. ${ }^{39}$ The ratio can be calculated using the following formula:

Incremental cost - effectiveness ratio $($ ICER $)=\frac{\text { Incremental cost }}{\text { Incremental Effect }}$

Incremental cost is the difference of average costs of intervention and comparator, whereas the incremental effect is the difference of average DALYs of intervention and comparator. The decision tree for the economic evaluation is shown in figure 1 . The probabilities to estimate the average cost per person and average DALYs averted per person are detailed in table 3. These probabilities were calculated from the survey data. Estimates show that probabilities of having oral health complications during the pregnancy period varied across different groups. IG1 group (MIOH) has the highest probability $(58 \%)$ of having an oral health problem followed by CG and IG2 (MIOH-DS) group. The probability of having timely treatment (the period that the treatment was provided

\begin{tabular}{|c|c|c|c|c|c|c|c|}
\hline & \multicolumn{2}{|c|}{ Control $(n=168)$} & \multicolumn{2}{|c|}{ MIOH (n=152) } & \multicolumn{2}{|c|}{ MIOH-DS (n=156) } & \multirow[b]{2}{*}{$P$ value } \\
\hline & $\mathbf{n}$ & $\%$ & $\mathbf{n}$ & $\%$ & $\mathbf{n}$ & $\%$ & \\
\hline \multicolumn{8}{|l|}{ Demographic } \\
\hline Age (mean, SD) & \multicolumn{2}{|c|}{$28.9(5.4)$} & \multicolumn{2}{|c|}{$29.6(5.5)$} & \multicolumn{2}{|c|}{$29.3(8.1)$} & 0.60 \\
\hline Country of Origin, Australia* & 108 & 64.3 & 94 & 61.4 & 89 & 59.3 & 0.66 \\
\hline Main language, English* & 113 & 66.9 & 100 & 64.9 & 103 & 67.8 & 0.87 \\
\hline Tertiary education $†$ & 87 & 52.4 & 89 & 58.9 & 81 & 54.7 & 0.51 \\
\hline Marital status, single* & 51 & 30.9 & 46 & 30.3 & 29 & 19.2 & 0.06 \\
\hline Employment, not working* & 78 & 47.3 & 77 & 50.7 & 87 & 57.6 & 0.18 \\
\hline Smoking $^{*}$ & 24 & 14.2 & 16 & 10.6 & 15 & 9.9 & 0.44 \\
\hline Other substance use ${ }^{*}$ & 2 & 1.2 & 0 & 0 & 1 & 0.7 & 0.41 \\
\hline Private health insurance ${ }^{*}$ & 31 & 18.6 & 23 & 15.2 & 32 & 21.6 & 0.36 \\
\hline Pension/healthcare card* & 63 & 37.3 & 61 & 40.4 & 51 & 34 & 0.52 \\
\hline \multicolumn{8}{|l|}{ Dental } \\
\hline $\begin{array}{l}\text { Do you currently have any } \\
\text { problems or concerns with your } \\
\text { teeth, gums or mouth? yes }\end{array}$ & 87 & 51.8 & 88 & 57.5 & 70 & 46.7 & 0.17 \\
\hline $\begin{array}{l}\text { Have you received any } \\
\text { information about 'oral healthcare } \\
\text { during pregnancy'? yes* }\end{array}$ & 16 & 9.6 & 13 & 8.5 & 9 & 6 & 0.48 \\
\hline $\begin{array}{l}\text { Have you seen a dentist in the } \\
\text { previous } 12 \text { months, yes }{ }^{*}\end{array}$ & 62 & 36.7 & 50 & 32.7 & 49 & 32.7 & 0.44 \\
\hline
\end{tabular}

${ }^{*}$ Contains missing values $(<10)$.

†Contains missing values $(=10)$.

$\mathrm{MIOH}-\mathrm{DS}$, Midwifery Initiated Oral Health-Dental Service. 


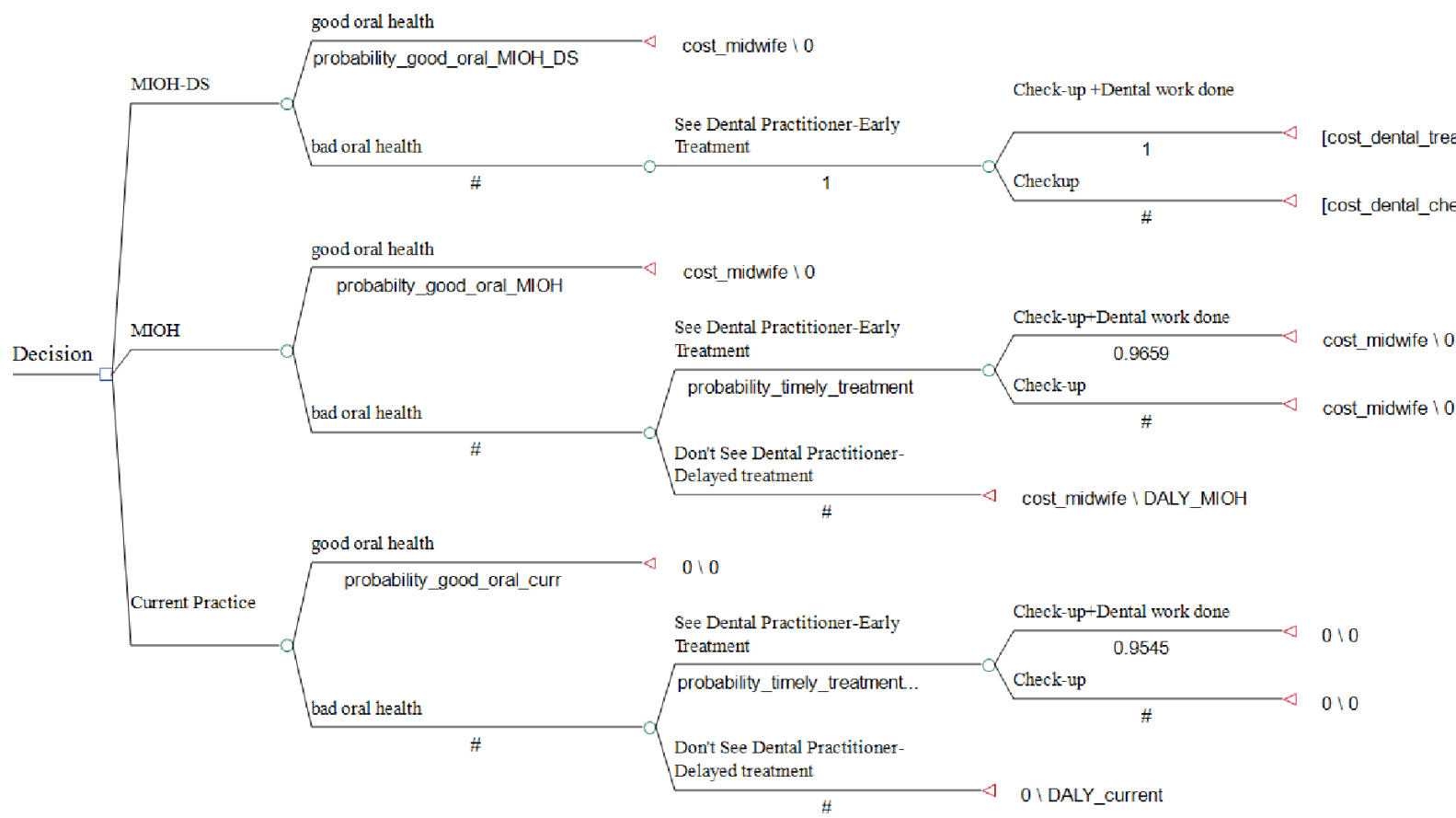

Figure 1 Decision tree of cost-effectiveness analysis of oral health intervention. DALY, Disability Adjusted Life Year; MIOH-DS, Midwifery Initiated Oral Health-Dental Service.

to the women by dentists that were part of the intervention programme or private practitioners referred by the programme) given that women had oral health problem is 1 for IG2 group because of the design of the programme. In other words, everyone in the IG2 group was treated if they had any oral health problem during the pregnancy period. However, probabilities were different for the other two groups. IG1 group had a probability of $27 \%$ of visiting any dentist whereas it was $20 \%$ for the CG based on trial data ${ }^{23}$ (table 3). Once patients visited dental facilities, they could have either a dental check-up or a dental check-up with necessary treatment. This was applicable for all groups. It was found that, everyone in IG2 group had both dental check-up and necessary dental work done. Not everyone in the IG1 group and control group had both dental check-up and treatment done, although the probability is low. ${ }^{23}$

Using the costs, DALYs and probabilities, the average per-person cost and average per-person DALYs were estimated. Since these costs are calculated from the

Table 3 Input baseline parameter and sensitivity analysis

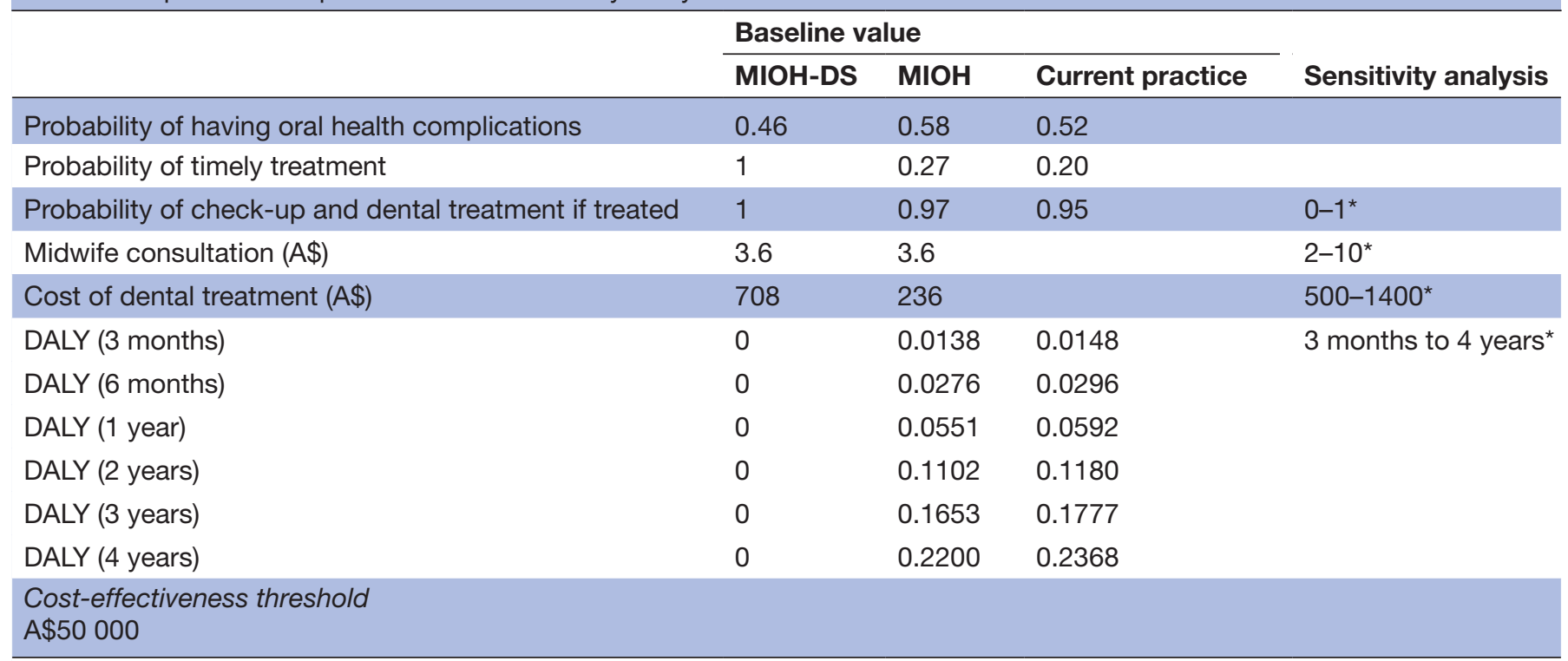

*Author assumption.

DALY, Disability Adjusted Life Year; MIOH-DS, Midwifery Initiated Oral Health-Dental Service. 
Table 4 Incremental cost-effectiveness ratio

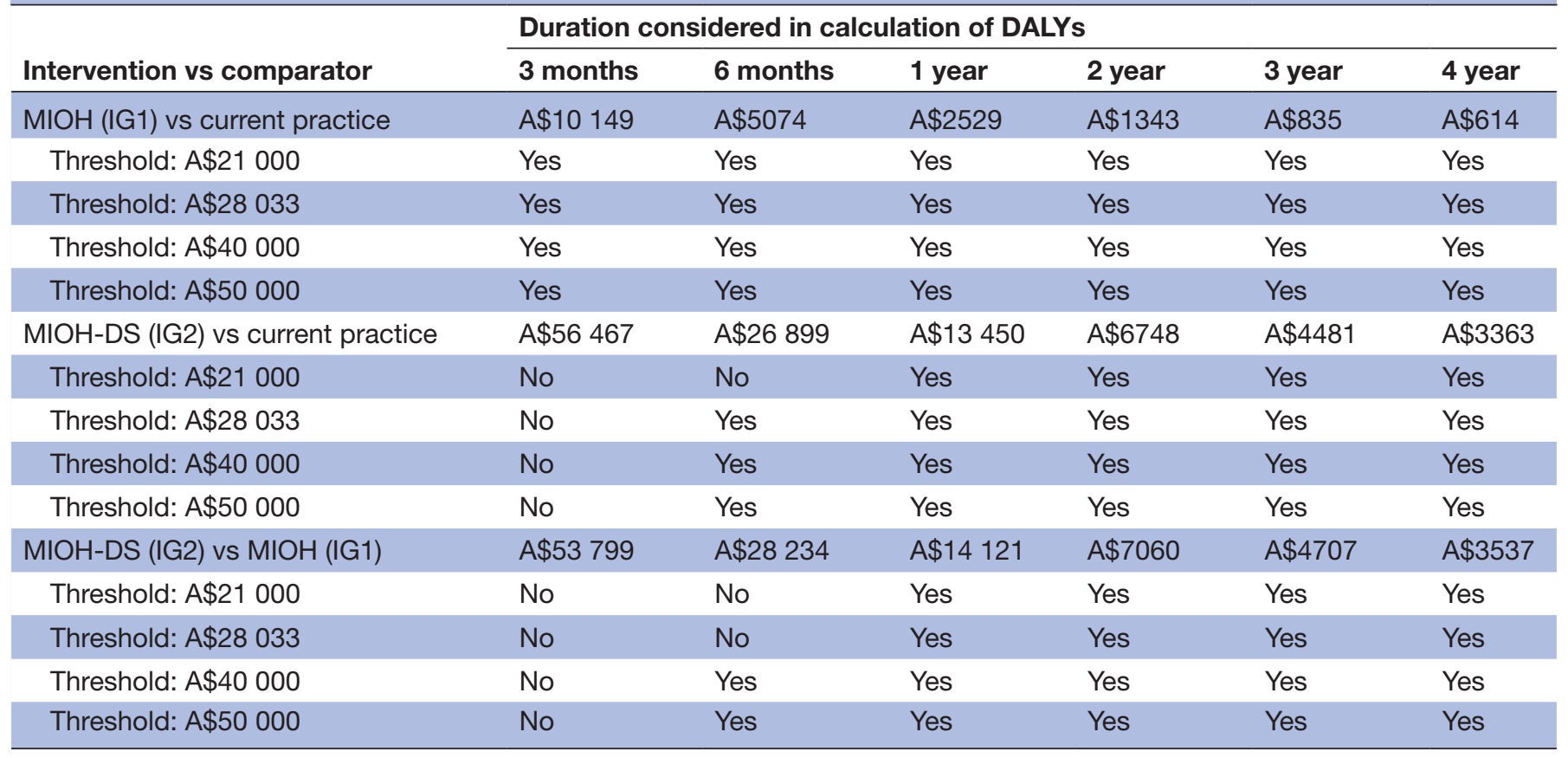

DALY, Disability Adjusted Life Year; IG, Intervention Group; MIOH-DS, Midwifery Initiated Oral Health-Dental Service.

public healthcare provider perspective, there exists no cost for the current practice which is no intervention. It is also assumed that the intervention is given once for all different scenarios where DALYs are calculated for 3 months, 6 months, 1-year, 2-year, 3-year and 4-year periods. Therefore, the average costs do not vary across scenarios.

On the effectiveness side, it was assumed that having no oral health problems would be assigned a DALY weight of zero. Further, that treatment completely cures the person from oral health problem, which will also result in a DALY weight of zero. As the interest is in the difference in the effect, assigning zero value to a problem free state will not affect our estimation. Therefore, the average per person DALYs for women from IG2 was zero. Again the average per person DALYs for IG1 is lower than the control group in all scenarios as shown in the table 3.

In table 4, the ICER is shown for different scenarios for different sets of interventions and comparators. The threshold is shown in table 4 which is based on WHO and Australian guidelines. ${ }^{2240}$ Thresholds of different years are used for different scenarios as different durations of the DALYs are used in this study. According to WHO guidelines, the intervention is 'highly cost-effective' if ICER is lower than GDP per capita of the country. As shown in table 4, the MIOH intervention IG1 is cost-effective in all scenarios considered in the study when compared with the current practice. The same table shows that MIOH-DS IG2 is also cost-effective in all scenarios with current practice being the comparator, except the 3-month scenarios, and the 6-month scenario with $A \$ 21000$ threshold. The comparator remains the same in this case as well. Similarly, MIOH-DS IG2 is cost-effective in all scenarios compared with MIOH IG1 (comparator), except when using all the Australian threshold for the 3 months scenario and A $\$ 21$ 000 and $A \$ 28234$ thresholds for the 6 months scenario.

Figure 2 shows the sensitivity of ICER (MIOH-DS vs current practice) with respect to input parameters of the model. The results are presented for two scenarios based on which the other scenarios can be inferred. As can be seen from the figure 2 that the ICER is not sensitive to the cost of dental treatment and cost of midwife for both scenarios. However, the ICER is sensitive to the probability of timely treatment of current practice in cases where the effect of the intervention remains for 6 months. But when the effect of the intervention is 1 year, the maximum value of ICER remains below the threshold of A $\$ 50000$. Therefore, the ICER does not exceed the A $\$ 50000$ threshold of willingness-to-pay if the effect of the intervention is valid for more than 1 year. Even in the 3 months scenario the ICER does not exceed the threshold if the probability of timely treatment is lower than 0.57 . Moreover, the ICER exceeds the threshold by some margin if the upper bound of cost of dental treatment is considered $90 \%$ higher than value used in the modelling in the 6 months scenario.

Similar results were found when the input parameters are varied in the model with MIOH-DS IG2 as the intervention and MIOH IG1 as the comparator. The sensitivity of the ICER is presented in figure 3 which shows that the model is not sensitive to cost of dental treatment except the probability of timely treatment in case of $\mathrm{MIOH}$ IG2. As previously stated, the ICER is not sensitive to the probability of timely treatment when the duration of the effect is 1 year or more. Further, the sensitivity analysis of the model of $\mathrm{MIOH}$-current practice is also considered (figure 4). The cost-effectiveness remained unchanged 


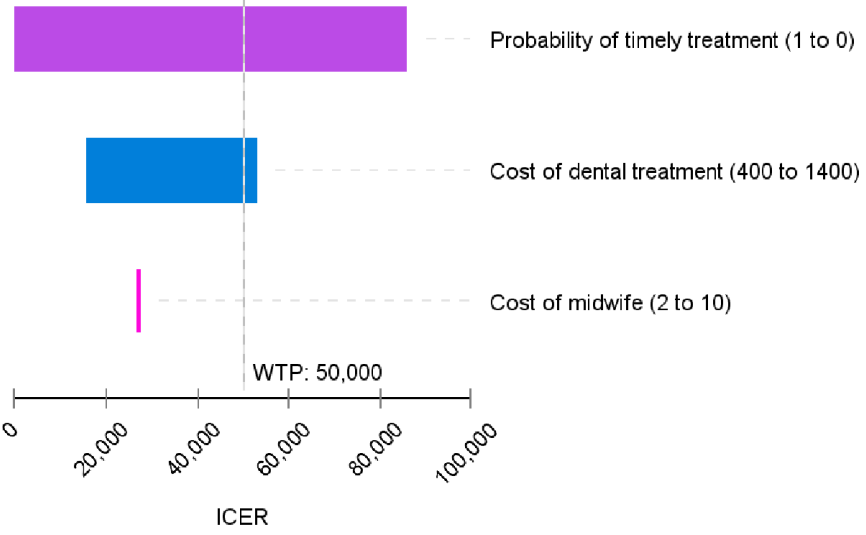

(a) duration: 1 year

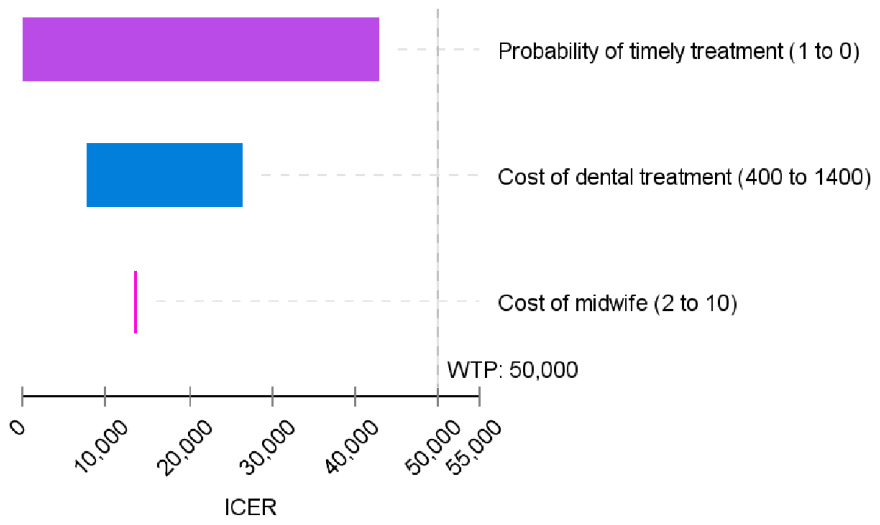

(a) duration: 2 year

Figure 2 One-way sensitivity analysis of cost-effectiveness of oral health intervention (Midwifery Initiated Oral HealthDental Service versus current practice). ICER, incremental cost-effectiveness ratio; WTP, willingness-to-pay.

for all scenarios when the cost of a midwife and probability of timely treatment varied. The sensitivity analysis for 3 months scenario was not considered since the intervention was not cost-effective in those scenarios for all the modellings. The duration used ranged from 3 months to 4 years with testing using these periods. This follows direction provided by dental clinical specialists on the duration of effectiveness of treatment for ideal patients ranging from 1 year to lifetime. In our $\mathrm{MIOH}$ sample, the most common treatments needed and provided were dental prophylaxis (removal of plaque/stain and calculus for $100 \%$ ), restorations (for dental decay for 100\%) and extractions (for 8\%). This was undertaken to provide an infection free and functional oral cavity as per the study protocol. ${ }^{21}$ For low-risk patients, the follow-up period post dental prophylaxis is generally 1 to 2 years, ${ }^{41}$ restorations is 2 years ${ }^{42}$ and uncomplicated extractions do not require any additional follow-up.

\section{DISCUSSION}

Pregnancy usually results in considerable changes in the oral health of women, which may lead to gum infections

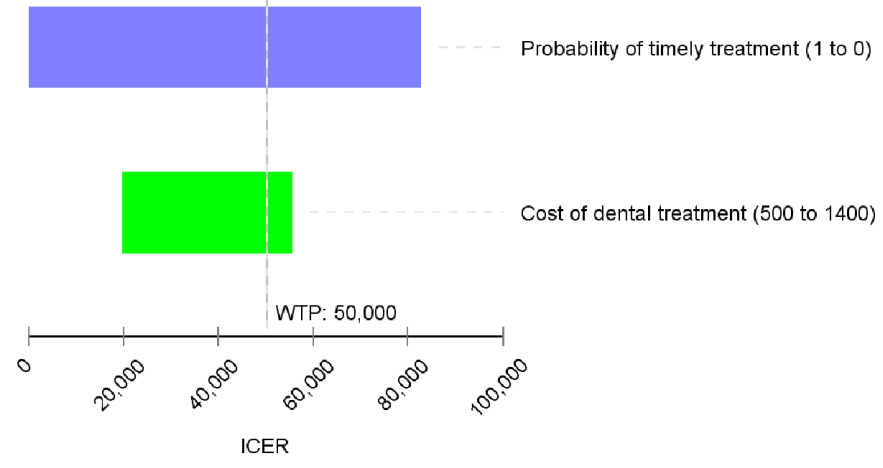

(a) duration: 1 year

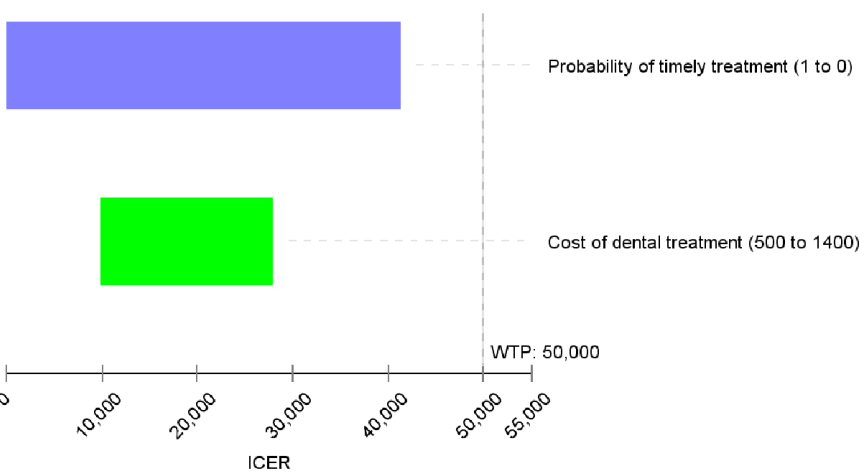

(a) duration: 2 year

Figure 3 One-way sensitivity analysis of cost-effectiveness of oral health intervention (MIOH-DS vs $\mathrm{MIOH}$ ). ICER, incremental cost-effectiveness ratio; MIOH-DS; Midwifery Initiated Oral Health-Dental Service; WTP, willingness-to-pay.

and tooth decay. The delivery of routine dental care during this period has the potential to reduce gum disease and dental decay, while supporting the best oral health for the newborn infant. The MIOH-DS randomised controlled trial conducted from 2012 to 2015 recruited 638 pregnant women attending antenatal clinics in three major public health services in Sydney. Improvements in the use of dental services were found

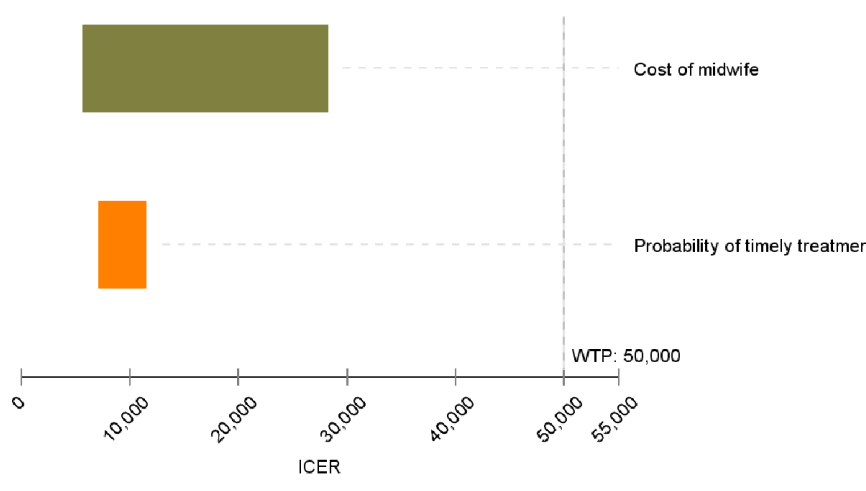

(a) duration: 1 year

Figure 4 One-way sensitivity analysis of cost-effectiveness of oral health intervention (Midwifery Initiated Oral Health vs current). ICER, incremental cost-effectiveness ratio; WTP, willingness-to-pay. 
in the midwifery only intervention (28\%) (IG1), and the combined midwife and dentist intervention group $(87 \%)$ (IG2), and to a lesser extent in the CG (20\%). All groups who attended the dentist noted a self-reported dental issue with no significant differences across groups. ${ }^{23}$ All three groups improved their dental knowledge and self-reported quality of oral health, ${ }^{23}$ although only the MIOH-DS (midwife and public dentist combined intervention) resulted in significant reductions in oral illhealth outcomes (sulcus bleeding index, clinical gum attachment loss, plaque index, decayed teeth, with increased filled teeth). The availability of this free dental service combined with midwife assessment and referral, has improved the oral health of this large sample of pregnant Australian women.

The cost-effectiveness analysis found that, compared with current practice, the MIOH-DS programme and $\mathrm{MIOH}$ comparator represent cost-effective interventions options for improving the oral health outcomes of pregnant women in Australia. Our economic evaluation supports the benefits of these interventions across longer time scales. It is noteworthy that the cost difference between the midwife only intervention and the combined intervention is substantial. However, for the midwife only intervention, only improvements in knowledge and selfreported quality of oral health were demonstrated. For the objectively determined oral health outcomes to be achieved, the full MIOH-DS intervention is required and thus both components of the programme need to be readily accessible before it can be scaled up across Australia.

Currently the MIOH training programme is available for all midwives to complete online through the Australian College of Midwives ${ }^{2543}$ The programme is endorsed as a continuing professional development (CPD) activity (16 CPD points) and therefore provides an added incentive for midwives to undertake the training and gain knowledge and confidence to promote oral health. Over 250 midwives have been trained in Victoria. ${ }^{44}$ Dental Health Services Victoria are also evaluating the training of those midwives whom they sponsor to undertake the training. ${ }^{45}$

The dental care component though, is currently not accessible for all pregnant women in Australia. This is in spite of Australia's National Oral Health Plan recognising the importance of pregnancy as a key life stage for oral health and the need to involve other health professionals in oral health programmes. ${ }^{46}$ Affordable dental care is available only for those in lower socioeconomic groups through public dental services and the waiting times to access services can vary depending on the state. ${ }^{47}$ Australia's National Oral Health Plan also includes access guidelines for oral healthcare which include pregnancy and the role for midwives. ${ }^{46}$ The potential delay in accessing public dental services for pregnant women is of concern as the second trimester is the ideal period to undertake any dental treatment. ${ }^{48}$ Exacerbating the situation is the fact that many mothers from disadvantaged groups tend to access antenatal services later in pregnancy ${ }^{49}$ and thus would have further delays in receiving public dental care through MIOH-DS referrals.

One way of addressing this in Australia could be to offer eligible pregnant women priority access to public dental services without a waiting list. This is a strategy which has been implemented in the state of Victoria through a minimal co-payment system. ${ }^{36}$ This strategy was found to be effective in scaling up the MIOH-DS programme in Victoria with more than a $50 \%$ increase in pregnant women accessing Public Dental Services. ${ }^{44}$ These positive results from Victoria, combined with the fact that $35 \%-40 \%$ of participants in each group of the MIOH-DS $\mathrm{trial}^{23}$ were eligible for public dental services, suggest that a priority referral pathway could benefit all Australian pregnant women from low socioeconomic backgrounds.

To benefit more pregnant women across Australia, a government (Medicare) funded programme may need to be explored to provide care through both the public and private dental systems. Such an approach is being adopted in Australia to improve early childhood oral health through the Medicare Child Dental Benefit Schedule $^{50}$ which provides eligible children up to $\mathrm{A} \$ 1000$ over 2 years to receive basic dental care. Although this benefit is means-tested, the eligibility criteria is not as stringent as those to access public dental services and thus offering a similar scheme to pregnant women would benefit greater numbers of families across Australia. The added benefit of such a scheme is that it would reduce the burden on the public dental services which employs only a small proportion $(5.7 \%)$ of dentists in Australia. ${ }^{51}$ The last option would be to provide universal access to basic dental care to all pregnant women in Australia similar to the UK system where all mothers receive free dental care during pregnancy and for 12 months after giving birth through the National Health Service which uses private practitioners. ${ }^{52}$ Universal access though would heavily depend on substantial investment from both the Federal and State health authorities in Australia.

To better understand the economic implications of implementing the MIOH or MIOH-DS programme it is important to assess the potential costing. For the $\mathrm{MIOH}$ programme this includes training costs for the midwives and ongoing programme costs. The number of midwives that worked in the Australian public system was 21149 in $2017,{ }^{53}$ the training costs for this workforce will be $\mathrm{A} \$ 17.6$ million. Each year, 1200 new midwives join the Australian public health system annually which would translate into an additional training costs of about $\mathrm{A} \$ 1$ million every year for the midwives in $\mathrm{MIOH}$. The total number of births in Australia averages 300000 per year and $75 \%$ of all births takes place in public hospital, therefore the $\mathrm{MIOH}$ ongoing cost will be $\mathrm{A} \$ 810000$. The total costs of MIOH programme in the first year of implementation nationally will be $\mathrm{A} \$ 18.5$ million. After the first year, the costs for MIOH delivery and training of new midwives will be approximately A $\$ 2$ million per year.

The MIOH-DS programme that involves the dental clinician and treatment is estimated to cost at $\mathrm{A} \$ 81.5$ 
million on an ongoing basis. This includes the total cost from $\mathrm{MIOH}$ on ongoing basis plus the dental check-up and treatments' costs assuming the proportion of women with poor oral health nationally is similar to the sample group. In the first implementation year and inclusive of the training of all of the midwives nationally, the estimated total cost is $\mathrm{A} \$ 99$ million, about $0.1 \%$ of the total public health expenditure in Australia. ${ }^{54}$

Based on the costings and the fact that the $\mathrm{MIOH}$ programme was cost-effective in all scenarios compared with current practice, we suggest that this model of care might be a more feasible option for policymakers in countries that have limited resources to support affordable and accessible dental care for pregnant women. Increased oral health knowledge could translate to improved preventative behaviours and oral health status. ${ }^{55}$ The $\mathrm{MIOH}$ programme would also be best suited for countries that already have systems in place that offer pregnant women priority access to public dental care such a UK and Poland. ${ }^{52} 56$

Finally, it is important to note that midwives play an important role in health promotion and preventative strategies. Midwives are also highly effective in providing public heath messaging and this part of their role has expanded substantially in the past decade ${ }^{57}$ If this aspect of their role is to be further expanded consideration will need to be given to already heavy workloads and fragmented models of care in order to support midwives as well as women and enable continued positive health outcomes and sustainability into the future.

There are limitations to this study. First, DALYs were used as a measure of effectiveness in the absence of Quality Adjusted Life Year (QALY) in the study. The QALY would have reflected a better measurement of effectiveness which could affect the findings of the study. Second, costings used was for the spent during the intervention period with recognition that the benefits of oral treatment extended beyond the project scope period, up to 4 years post. Ideally, longitudinal data on oral health costs and benefits would be collected over time on the participants. Third, the economic evaluation is static in nature in the absence of transitional probabilities among states of health status. Fourth, the duration of different oral conditions was not known with assumptions made. Oral complications during the pregnancy with shorter duration can nullify the effectiveness of the interventions. Ideally, to determine the effectiveness of the oral health treatment to the participants would entail collection of data on at least annually for a number of years. However, the practicality of conducting a longitudinal study of this type was beyond the scope of this research. Thereby, oral health clinician directions were obtained together with sensitivity analysis, providing an average effectiveness period of 4 years. Fifth, the study findings are sensitive to the probability of timely treatment, and finally, the study did not consider any health benefits for the children following birth as these data were not available. The study time frame was limited given the funding source.
Ideally, the analysis would have benefited from longitudinal follow-up on the pregnant women, the infants born and other children or adults in the household to assess ongoing effect of the programme. Despite the limitations, it is the first study of its kind to evaluate such an oral health intervention in Australia. Moreover, different scenario analyses were conducted to overcome the lack of information on the duration of oral health problems and the findings are robust for other inputs of the model.

\section{CONCLUSIONS}

This economic evaluation has demonstrated that the midwives' low-cost intervention providing oral health education, assessment and referral to existing dental services was cost-effective, although it did not deliver an oral health improvement. However, the Midwives' and public dentists' combined interventions were costeffective when the benefits were considered over a longer time period. The $\mathrm{MIOH}$ and MIOH-DS cost-effectiveness study supports further funding to deliver this programme to all pregnant women. Substantial investment from state or Commonwealth governments is required along with consideration of midwives workload in order to deliver the long-term benefits in oral health. This could be achieved through publicly-funded dental services or through a funded fee for service private-public partnership based model of service delivery for socioeconomically disadvantaged women during pregnancy made available at Australian antenatal clinics.

\section{Author affiliations}

${ }^{1}$ Translational Health Research Institute, Digital Health Cooperative Research Centre, Economics, Finance and Property, School of Business, Western Sydney University, Penrith, New South Wales, Australia

${ }^{2}$ Centre for Oral Health Outcomes \& Research Translation (COHORT), Western Sydney University, South Western Sydney Local Health District, Ingham Institute Applied Medical Research, Liverpool, New South Wales, Australia

${ }^{3}$ School of Dentistry, Faculty of Medicine and Health, University of Sydney, Camperdown, New South Wales, Australia

${ }^{4}$ Translational Health Research Institute, Western Sydney University, Penrith, New South Wales, Australia

${ }^{5}$ School of Nursing and Midwifery, Western Sydney University, Penrith, New South Wales, Australia

${ }^{6}$ Ingham Institute for Applied Medical Research, Liverpool, New South Wales, Australia

${ }^{7}$ Poche Centre for Indigenous Health, The University of Sydney, Sydney, New South Wales, Australia

${ }^{8}$ Oral Health Services and Sydney Dental Hospital, Sydney Local Health District, Surry Hills, New South Wales, Australia

${ }^{9}$ Oral Health Services, Nepean Blue Mountains Local Health District, Penrith, New South Wales, Australia

${ }^{10}$ Oral Health Services, South Western Sydney Local Health District, Liverpool, New South Wales, Australia

${ }^{11}$ Australian Catholic University, North Sydney, New South Wales, Australia

Twitter Ajesh George @ajeshgeorge_and Hannah G Dahlen @hannahdahlen

Acknowledgements The support of the midwifery and dental staff involved in the MIOH-DS trial across South Western Sydney Local Health District and Nepean Blue Mountains Local Health District is gratefully acknowledged.

Contributors AG, KWT and MJ were responsible for the study conception and design, KWT and MA organised the data and performed the data analysis. KWT, AG, 
MJ and MA were responsible for drafting the manuscript. AB, HGD, JS, SA, SB, AY and RS made critical revisions to the paper for important intellectual content. All authors read and approved of the final manuscript.

Funding This work was supported by the National Health and Medical Research Council project grant (APP1022007).

Competing interests None declared.

Patient and public involvement Patients and/or the public were not involved in the design, or conduct, or reporting, or dissemination plans of this research.

Patient consent for publication Not required.

Ethics approval Ethics approval was obtained from the Human Research Ethics Committees of Sydney Local Health District (HREC11/CRGH/289) and the University of Western Sydney (H9709).

Provenance and peer review Not commissioned; externally peer reviewed.

Data availability statement All data relevant to the study are included in the article or uploaded as supplementary information.

Open access This is an open access article distributed in accordance with the Creative Commons Attribution Non Commercial (CC BY-NC 4.0) license, which permits others to distribute, remix, adapt, build upon this work non-commercially, and license their derivative works on different terms, provided the original work is properly cited, appropriate credit is given, any changes made indicated, and the use is non-commercial. See: http://creativecommons.org/licenses/by-nc/4.0/.

\section{ORCID iDs}

Kathy W Tannous http://orcid.org/0000-0002-3153-5652

Ajesh George http://orcid.org/0000-0002-6795-2546

Moin Uddin Ahmed http://orcid.org/0000-0002-1733-5216

Hannah G Dahlen http://orcid.org/0000-0002-4450-3078

John Skinner http://orcid.org/0000-0002-4866-053X

Maree Johnson http://orcid.org/0000-0001-6653-3780

\section{REFERENCES}

1 American Academy of Pediatrics, The American College of Obstetricians and Gynecologists. Guidelines for perinatal care. 7th ed, 2012.

2 Silk H, Douglass AB, Douglass JM, et al. Oral health during pregnancy. Am Fam Physician 2008;77:1139-44.

3 Romero R, Gotsch F, Pineles B, et al. Inflammation in pregnancy: its roles in reproductive physiology, obstetrical complications, and fetal injury. Nutr Rev 2007;65:194-202.

4 Çolak H, Dülgergil CT, Dalli M, et al. Early childhood caries update: a review of causes, diagnoses, and treatments. J Nat Sci Biol Med 2013;4:29-38.

5 Meyer F, Enax J. Early childhood caries: epidemiology, aetiology, and prevention. Int J Dent 2018;2018:1-7.

6 George A, Sousa MS, Kong AC, et al. Effectiveness of preventive dental programs offered to mothers by non-dental professionals to control early childhood dental caries: a review. BMC Oral Health 2019;19:172.

7 Gussy M, Ashbolt R, Carpenter L, et al. Natural history of dental caries in very young Australian children. Int J Paediatr Dent 2016;26:173-83.

8 Keirse MJNC, Plutzer K. Women's attitudes to and perceptions of oral health and dental care during pregnancy. J Perinat Med 2010;38:3-8.

9 George A, Johnson M, Blinkhorn A, et al. Views of pregnant women in South western Sydney towards dental care and an oral-health program initiated by midwives. Health Promot J Austr 2013;24:178-84.

10 George A, Johnson M, Blinkhorn A, et al. The oral health status, practices and knowledge of pregnant women in south-western Sydney. Aust Dent J 2013;58:26-33.

11 George A, Johnson M, Blinkhorn A, et al. Promoting oral health during pregnancy: current evidence and implications for Australian midwives. J Clin Nurs 2010;19:3324-33.

12 Centre for Oral Health Strategy NSW. Oral health 2020: a strategic framework for dental health in NSW. North Sydney: NSW Ministry of Health, 2013.

13 AlHW: Chrisopoulos S HJEA. Oral health and dental care in Australia: key facts and figures 2015. cat. no.DEN 229. Canberra: Australian Institute of Health and Welfare, 2016.
14 National Advisory Council on Dental Health. Report of the National Advisory Council on dental health. Canberra: Department of Health, 2012.

15 AlHW. A discussion of public dental waiting times information in Australia: 2013-14 to 2016-17. cat. no.DEN 230. Canberra: Australian Institute of Health and Welfare, 2018.

16 Victoria DHS. Healthy teeth healthy pregnancy [Internet], 2014. Available: www.dhsv.org.au/_data/assets/pdf_file/0008/31787/ Pregnancy-factsheet.pdf

17 NSW Health. Priority oral health program and list management protocols [Internet], 2008. Available: http://www1.health.nsw.gov.au/ pds/ActivePDSDocuments/PD2008 056.pdf

18 Thomas NJ, Middleton PF, Crowther CA. Oral and dental health care practices in pregnant women in Australia: a postnatal survey. BMC Pregnancy Childbirth 2008;8:13.

19 George A, Dahlen HG, Reath J, et al. What do antenatal care providers understand and do about oral health care during pregnancy: a cross-sectional survey in New South Wales, Australia. BMC Pregnancy Childbirth 2016;16:382.

20 George A, Johnson M, Duff M, et al. Maintaining oral health during pregnancy: perceptions of midwives in Southwest Sydney. Collegian 2011;18:71-9.

21 Johnson M, George A, Dahlen $\mathrm{H}$, et al. The midwifery initiated oral health-dental service protocol: an intervention to improve oral health outcomes for pregnant women. BMC Oral Health 2015;15:2.

22 ten Hoope-Bender P, de Bernis L, Campbell J, et al. Improvement of maternal and newborn health through midwifery. Lancet 2014;384:1226-35.

23 George A, Dahlen HG, Blinkhorn A, et al. Evaluation of a midwifery initiated oral health-dental service program to improve oral health and birth outcomes for pregnant women: a multi-centre randomised controlled trial. Int J Nurs Stud 2018;82:49-57.

24 George A, Dahlen HG, Blinkhorn A, et al. Measuring oral health during pregnancy: sensitivity and specificity of a maternal oral screening (mos) tool. BMC Pregnancy Childbirth 2016;16:347.

25 George A, Lang G, Johnson M, et al. The evaluation of an oral health education program for midwives in Australia. Women Birth 2016;29:208-13.

26 Dahlen HG, Johnson M, Hoolsema J, et al. Process evaluation of the midwifery initiated oral health-dental service program: perceptions of midwives in greater western Sydney, Australia. Women Birth 2019;32:e159-65.

27 Ajwani S, Sousa MS, Villarosa AC, et al. Process evaluation of the midwifery initiated oral health-dental service program: perceptions of dental professionals. Health Promot J Austr 2019;30:333-43.

28 George A, Villarosa AR, Patterson Norrie T, et al. Process evaluation of the midwifery initiated oral health-dental service program: perceptions of pregnant women. Aust Dent J 2019;64:55-65.

29 AlHW. Health expenditure Australia 2017-18. cat. no.HWE 77. Canberra: Australian Institute of Health and Welfare, 2019.

30 Centers for Medicare \& Medicaid Services. National health expenditure data: historical [Internet]. Baltimore: CMS, 2020. https:// www.cms.gov/Research-Statistics-Data-and-Systems/StatisticsTrends-and-Reports/NationalHealthExpendData/NationalHealthAc countsHistorical

31 AlHW. Australia's health expenditure: an international comparison [Internet], 2019. Available: https://www.aihw.gov.au/getmedia/ ba3f6a4c-3059-4340-b1ca-b4ddd5630e4f/aihw-hwe-75.pdf.aspx? inline=true

32 Bernitz S, Aas E, Øian P. Economic evaluation of birth care in low-risk women. A comparison between a midwife-led birth unit and a standard obstetric unit within the same hospital in Norway. A randomised controlled trial. Midwifery 2012;28:591-9.

33 Tracy SK, Hartz D, Hall B, et al. A randomised controlled trial of caseload midwifery care: M@NGO (Midwives @ New Group practice Options). BMC Pregnancy Childbirth 2011;11:82.

34 Pagano E, De Rota B, Ferrando A, et al. An economic evaluation of water birth: the cost-effectiveness of mother well-being. J Eval Clin Pract 2010;16:916-9.

35 George A, Kong A, Sousa M, et al. Long-Term effectiveness of the midwifery initiated oral Health-Dental service program on maternal oral health knowledge, preventative dental behaviours and the oral health status of children in Australia 2021.

36 Brennan DS, Spencer AJ. Disability weights for the burden of oral disease in South Australia. Popul Health Metr 2004;2:7.

37 NSW Health. Oral health fee for service schedule of fees 2016 [Internet], 2016. Available: https://www1.health.nsw.gov.au/pds/ ActivePDSDocuments/IB2016_023.pdf

38 Pharmaceutical Benefits Advisory Committee. Guidelines for preparing submissions to the pharmaceutical benefits Advisory Committee (version 5.0). Canberra: PBAC, 2016. 
39 Edney LC, Haji Ali Afzali H, Cheng TC, et al. Estimating the reference incremental cost-effectiveness ratio for the Australian health system. Pharmacoeconomics 2018;36:239-52.

40 Hutubessy R, Chisholm D, Edejer TT-T. Generalized costeffectiveness analysis for national-level priority-setting in the health sector. Cost Eff Resour Alloc 2003;1:8.

41 Lamont T, Worthington HV, Clarkson JE, et al. Routine scale and Polish for periodontal health in adults. Cochrane Database Syst Rev 2018;12:CD004625.

42 Fee PA, Riley P, Worthington HV, et al. Recall intervals for oral health in primary care patients. Cochrane Database Syst Rev 2020;10:CD004346.

43 Australian College of Midwives. Midwifery initiated oral health [Internet. Available: https://www.midwives.org.au/shop/midwiferyinitiated-oral-health

44 Dental Health Services Victoria. Is $\mathrm{MIOH}$ influencing the numbers of pregnant women accessing public dental services? [Internet. Melbourne: DHSV, 2018. https://www.dhsv.org.au/_data/assets/ pdf_file/0004/87124/MIOH-Newsletter-Final-_-2018-06-25.pdf

45 Dental Health Services Victoria. Online midwifery initiated oral health education program [Internet]. Melbourne: DHSV. https://www.dhsv. org.au/oral-health-programs/hfhs/eoi

46 COAG Health Council. Healthy mouths healthy lives. Australia's National Oral Health Plan 2015-2024 [Internet, 2015. http://www. coaghealthcouncil.gov.au/Portals/0/Australia\%27s\%20National\% 20Oral\%20Health\%20Plan\%202015-2024_uploaded\%20170216.pdf

47 Department of Health. The dental system [Internet], 2012. Available: https://www1.health.gov.au/internet/publications/publishing.nsf/ Content/report_nacdh report_nacdh_ch2 report_nacdh_ds

48 Council on Clinical Affairs. Guideline on perinatal oral health care. American Academy of Pediatric Dentistry 2009;37:15-16.
49 Department of Health. Antenatal visits [Internet], 2020. Available: https://www.health.gov.au/resources/pregnancy-care-guidelines/ part-b-core-practices-in-pregnancy-care/antenatal-visits

50 Department of Health. The child dental benefits schedule [Internet], 2018. Available: https://www1.health.gov.au/internet/main/ publishing.nsf/Content/childdental

51 AlHW. Oral health and dental care in Australia [Internet], 2019. Available: https://www.aihw.gov.au/reports/dental-oral-health/oralhealth-and-dental-care-in-australia/contents/dental-workforce

52 NHS. Are pregnant women entitled to free NHS dental treatment? [Internet]. NHS, 2019. https://www.nhs.uk/common-healthquestions/pregnancy/are-pregnant-women-entitled-to-free-nhsdental-treatment

53 Department of Health. Australia's future health workforce report - midwives. Canberra: Commonwealth Government of Australia, 2019.

54 Phillips J, Grove A, Cook L. Budget review 2019-20 index [Internet. Canberra: Commonwealth of Australia, 2019. https://www.aph.gov. au/About_Parliament/Parliamentary_Departments/Parliamentary_ Library/pubs/rp/BudgetReview201920/Health\#: :text=Total\% 20spending\%20on\%20health\%20in,expenditure\%20(see\% 20Table\%201

55 Brennan D, Spencer J, Roberts-Thomson K. Dental knowledge and oral health among middle-aged adults. Aust N Z J Public Health 2010;34:472-5

56 Biggs A. Dental reform: an overview of universal dental schemes. Social Policy Section: Parliament of Australia, 2012.

57 Sanders J, Hunter B, Warren L. A wall of information? Exploring the public health component of maternity care in England. Midwifery 2016;34:253-60. 